\title{
The Analysis of Several Models of Investment Value of Logistics Project Evaluation
}

\author{
Ke Qiu Cheng Zhou \\ School of International Business, Beijing Language and Culture University, Beijing 10083, China \\ Correspondence should be addressed to Ke Qiu Cheng Zhou; zhoukqc@yahoo.com.cn
}

Received 19 September 2012; Revised 19 December 2012; Accepted 17 February 2013

Academic Editor: Raouf Boucekkine

Copyright (C) 2013 Ke Qiu Cheng Zhou. This is an open access article distributed under the Creative Commons Attribution License, which permits unrestricted use, distribution, and reproduction in any medium, provided the original work is properly cited.

\begin{abstract}
The study of the logistics project evaluation model features reviews the traditional value evaluation model. On the basis of this, using the fuzzy theory, we establish several logistics project evaluation models under fuzzy environment. The analysis of the respective characteristics and the comparison of the calculated results of the three models show that these models are important methods of investment value of logistics evaluation.
\end{abstract}

\section{Introduction}

With the use of information technology and e-commerce and other modern technology, the investment in logistics industry has high uncertainty, irreversibility, and fuzziness. The application of NPV and other traditional methods of logistics project valuation is easy to cause the enterprise objective and actual value deviation. Giving full consideration to market volatility, uncertainty, irreversibility, and real option is of great practicality. The application of the real option to evaluate logistics project investment value is widely used. Schwartz and moon [1] believe that real option in venture capital evaluation can make better explanation. Dayanik [2] and so forth solved the one-dimensional diffusion process of optimal stopping problems, and the results for American option pricing, control, and so on are suitable. At the same time, in view of logistics project investment also having the characteristic of fuzziness, fuzzy factors with real option theory to carry on the research is very important. Carlsson and fullér [3] considered the rates of fuzzy-relation-fuzzy-option formula and used the optimization theory to build the project investment decision model of R\&D optimization. The fuzzy process, mixing process, and uncertain process proposed by Liu [4] can well explain the fuzzy financial market. Qin and $\mathrm{Li}$ [5] also presented the option pricing problem under fuzzy environment. Although most of the distribution function of a random variable can be obtained by statistical method, but in actual, because of the incomplete logistics project information and prior knowledge, we often fail to accurately collect and measure these data and cannot depict or control the various factors of the logistics project, which increase the logistics project management fuzzy. Thinking about these factors of the logistics project completely, many scholars are considering the classical option pricing theory on how to be improved. This paper reviews the logistics project evaluation model under traditional situation, then, on this foundation, it puts forward a few other value evaluation models and discusses and finally compares by numerical calculations.

\section{Traditional Logistics Project Evaluation Methods}

2.1. Method of $N P V$. From time $t$ to time $T$, we can get the net present value flow of the whole logistics project with investment opportunities at the time of $T$ :

$$
\Phi(t)=A(t) e^{-\alpha(T-t)}-I(t) e^{-\beta(T-t)}-I_{0},
$$

where $A(t)$ is logistics project asset value, $I(t)$ is the capital spending, where $\alpha$ is instantaneously the expected growth rate of $A(t), \beta$ is instantaneously expected growth rate of $I(t)$, and $I_{0}$ is the initial investment. 
2.2. A Kind of Simple Method about Net Present Value under the Random Environment. If in the risk neutral world, logistics project asset value $A(t)$ follows the stochastic differential equation (because of the uncertainty and other reasons of the logistics project investment, we can assume that the logistics project investment in capital spending $I(t)$ also follows the equation $[6,7])$ :

$$
\begin{gathered}
d A(t)=A(t) \alpha d t+A(t) \sigma_{1} d z_{1}, \\
d I(t)=I(t) \beta d t+I(t) \sigma_{2} d z_{2},
\end{gathered}
$$

where $\alpha$ is instantaneously the expected growth rate of $A(t)$, $\beta$ is instantaneously the expected growth rate of $I(t), \sigma_{1}$ is the instantaneous standard deviation of $A(t), \sigma_{2}$ is instantaneous standard deviation of $I(t), d z_{1}$ is the Wiener process of $A(t), d z_{2}$ is the Wiener process of $I(t)$, and the instantaneous correlation coefficient is $\rho_{12}$ : From time $t$ to time $T$, the logistics project investment opportunity to solve the problem and the threshold of implementation of investment are $\left(A^{*}, I^{*}, T\right)$; by McDonald and Siegel [6] and Dixit and Pindyck [7], we can get the formula of net present value flow:

$$
\Phi(t)=A(t) e^{-\alpha(T-t)} N\left(d_{1}\right)-I(t) e^{-\beta(T-t)} N\left(d_{2}\right)-I_{0}
$$

where

$$
\begin{aligned}
& d_{1}=\frac{\operatorname{In}(A(t) / I(t))+(\alpha-\beta)(T-t)}{\theta \sqrt{T-t}}+\frac{1}{2} \theta \sqrt{T-t} \\
& d_{2}=\frac{\operatorname{In}(A(t) / I(t))+(\alpha-\beta)(T-t)}{\theta \sqrt{T-t}}-\frac{1}{2} \theta \sqrt{T-t}
\end{aligned}
$$

where $\theta^{2}=\sigma_{1}^{2}-2 \rho_{12} \sigma_{1} \sigma_{2}+\sigma_{2}^{2}$ and $\rho_{12}$ is the relevant coefficient of the Wiener process $d z_{1}$ and $d z_{2}$.

\subsection{The Project Valuation Model of the Same Fluctuation}

Rate. In reality, the investment decision making of logistics project, often in the incomplete information condition, and their values have to be anticipated in order to determine the parameters. At the same time, the excessive pursuit of input variable accuracy of logistics project can lead to the output of results of fuzzy. From the view of fuzzy mathematics, logistics project value and the distribution of the cost of the total investment are a fuzzy number; according to the similarity of trapezoidal and normal distribution and mathematically convenient treatment, logistics project value can be expressed as a trapezoidal fuzzy number $V=\left[V_{1}, V_{2}, \alpha_{1}, \beta_{1}\right]$, and the total investment cost is $I=\left[I_{1}, I_{2}, \alpha_{2}, \beta_{2}\right]$. Trapezoidal fuzzy number $V$ lies in the interval $\left[V_{1}, V_{2}\right]$, most likely, $\left(V_{2}+\beta_{1}\right)$ and $\left(V_{1}-\alpha_{1}\right)$, respectively, which indicates the upper and lower bounds of the uncertain factor of the expected project profit cash flow. Similarly, the trapezoidal fuzzy number $I$ is the most likely value of the cost of logistics project investment, and it lies in the interval $\left[I_{1}, I_{2}\right],\left(I_{2}+\beta_{2}\right)$, and $\left(I_{1}-\alpha_{2}\right)$, respectively, which indicates the upper and lower bound of the expected investment cost. $T$ is the options of the expire time. According to Carlsson and Fullér [3], we obtained the model [8] based on the improved Merton formula evaluation under fuzzy environment:

$$
\begin{aligned}
\mathrm{FROV}= & V e^{-\delta T} N\left(d_{1}\right)-I e^{-r T} N\left(d_{2}\right) \\
= & {\left[V_{1}, V_{2}, \alpha_{1}, \beta_{1}\right] e^{-\delta T} N\left(d_{1}\right) } \\
& -\left[I_{1}, I_{2}, \alpha_{2}, \beta_{2}\right] e^{-r T} N\left(d_{2}\right) \\
= & \left(V_{1} e^{-\delta T} N\left(d_{1}\right)-I_{2} e^{-r T} N\left(d_{2}\right),\right. \\
& V_{2} e^{-\delta T} N\left(d_{1}\right)-I_{1} e^{-r T} N\left(d_{2}\right), \\
& \alpha_{1} e^{-\delta T} N\left(d_{1}\right)+\beta_{2} e^{-r T} N\left(d_{2}\right), \\
& \left.\beta_{1} e^{-\delta T} N\left(d_{1}\right)+\alpha_{2} e^{-r T} N\left(d_{2}\right)\right),
\end{aligned}
$$

where

$$
\begin{aligned}
& d_{1}=\frac{\operatorname{In}(E(V) / E(I))+\left(r+(1 / 2) \sigma^{2}\right) T}{\sigma \sqrt{T}}, \\
& d_{2}=\frac{\operatorname{In}(E(V) / E(I))+\left(r-(1 / 2) \sigma^{2}\right) T}{\sigma \sqrt{T}}, \\
& \sigma=\sqrt{\sigma_{1}^{2}+\sigma_{2}^{2}-2 \rho_{12} \sigma_{1} \sigma_{2}},
\end{aligned}
$$

where FROV is the real options value under fuzzy environment, $\sigma$ is the volatility, that is, the union fluctuation rate of the logistics project value and investment cost, $N(d)$ is the probability of less than $d$ under the condition of the standard normal distribution, $\sigma_{1}$ is the volatility of logistics project value, $\sigma_{2}$ is the volatility of the cost of the logistics project investment, and $\delta$ is the value loss during the process of option. The model provides a better method for us when we make evaluation for the same logistics project under fuzzy environment.

2.4. The Evaluation Model of Logistics Project Based on Compound Option Value. We combine the Geske model with trapezoidal fuzzy number combination, then we can get the Geske [9] model under fuzzy environment:

$$
\begin{aligned}
\text { FROV }= & {\left[V_{1}, V_{2}, \alpha_{1}, \beta_{1}\right] e^{-\delta t_{2}} N_{2}\left(a_{1}, b_{1} ; \sqrt{t_{2} / t_{1}}\right)-\left[I_{1}, I_{2}, \alpha_{2}, \beta_{2}\right] e^{-r t_{2}} N_{2}\left(a_{2}, b_{2} ; \sqrt{t_{1} / t_{2}}\right) } \\
& -\left[I_{1}^{\prime}, I_{2}^{\prime}, \alpha_{2}^{\prime}, \beta_{2}^{\prime}\right] e^{-r t_{1}} N_{1}\left(a_{2}\right)
\end{aligned}
$$




$$
=\left\{\begin{array}{c}
V_{1} e^{-\delta t_{2}} N_{2}\left(a_{1}, b_{1} ; \sqrt{t_{2} / t_{1}}\right)-I_{2} e^{-r t_{2}} N_{2}\left(a_{2}, b_{2} ; \sqrt{t_{1} / t_{2}}\right)-I_{2}^{\prime} e^{-r t_{1}} N_{1}\left(a_{2}\right), \\
V_{2} e^{-\delta t_{2}} N_{2}\left(a_{1}, b_{1} ; \sqrt{t_{2} / t_{1}}\right)-I_{1} e^{-r t_{2}} N_{2}\left(a_{2}, b_{2} ; \sqrt{t_{1} / t_{2}}\right)-I_{1}^{\prime} e^{-r t_{1}} N_{1}\left(a_{2}\right), \\
\alpha_{1} e^{-\delta t_{2}} N_{2}\left(a_{1}, b_{1} ; \sqrt{t_{2} / t_{1}}\right)+\beta_{2} e^{-r t_{2}} N_{2}\left(a_{2}, b_{2} ; \sqrt{t_{1} / t_{2}}\right)+\beta_{2}^{\prime} e^{-r t_{1}} N_{1}\left(a_{2}\right), \\
\beta_{1} e^{-\delta t_{2}} N_{2}\left(a_{1}, b_{1} ; \sqrt{t_{2} / t_{1}}\right)+\alpha_{2} e^{-r t_{2}} N_{2}\left(a_{2}, b_{2} ; \sqrt{t_{1} / t_{2}}\right)+\alpha_{2}^{\prime} e^{-r t_{1}} N_{1}\left(a_{2}\right)
\end{array}\right\},
$$

where

$$
\begin{aligned}
a_{1}= & {\left[V_{1}, V_{2}, \alpha_{1}, \beta_{1}\right] } \\
& \times \frac{\operatorname{In}\left(E\left(V_{0}\right) / E\left(V_{c}\right)\right)+\left(r-\delta+(1 / 2) \sigma^{2}\right) t_{1}}{\sigma \sqrt{t_{1}}}, \\
b_{1}= & {\left[V_{1}, V_{2}, \alpha_{1}, \beta_{1}\right] } \\
& \times \frac{\operatorname{In}\left(E\left(V_{0}\right) / E\left(I_{1}, I_{2}, \alpha_{2}, \beta_{2}\right)\right)+\left(r-\delta+(1 / 2) \sigma^{2}\right) t_{2}}{\sigma \sqrt{t_{2}}},
\end{aligned}
$$

where $a_{2}=a_{1}-\sigma \sqrt{t_{1}}, \quad b_{2}=b_{1}-\sigma \sqrt{t_{2}}$ and $V_{C_{1}}$ is the assets of the logistics project when a compound option should be performed, which can be concluded by the following equation:

$$
\begin{aligned}
& V_{C_{1} c} e^{-\delta\left(t_{2}-t_{1}\right)} N\left(c_{1}\right)-E\left(C_{3}\right) e^{-r\left(t_{2}-t_{1}\right)} N\left(c_{2}\right)-E\left(C_{2}\right)=0, \\
& c_{1}=\operatorname{In}\left(E\left(V_{c}\right) / E\left(I_{1}, I_{2}, \alpha_{2}, \beta_{2}\right)\right) \\
& \quad+\left(r-\delta+\frac{1}{2} \sigma^{2}\right)\left(t_{2}-t_{1}\right) \times\left(\sigma \sqrt{t_{2}-t_{1}}\right)^{-1}, \\
& c_{2}=c_{1}-\sigma \sqrt{t_{2}-t_{1}},
\end{aligned}
$$

where $\left[V_{1}, V_{2}, \alpha_{1}, \beta_{1}\right]$ is the present value of cash flows produced by the logistics project promotion, $\left[I_{1}, I_{2}, \alpha_{2}, \beta_{2}\right]$ is the investment required by the promotion of logistics project, $\left[I_{1}^{\prime}, I_{2}^{\prime}, \alpha_{2}^{\prime}, \beta_{2}^{\prime}\right]$ is the investment required by logistics project $\mathrm{R} \& \mathrm{D}$, and $E\left(V_{c}\right)$ is the critical value of the logistics project when the first buyer's option is closed, and it can be calculated by the Black-Scholes model. In $N_{2}\left(a_{1}, b_{1} ; \sqrt{t_{2} / t_{1}}\right)$, the first variable is less than $a_{1}$, the second variable is less than $b_{1}$, and two variable correlation coefficient is the probability function of the standard normal cumulative distribution of $\sqrt{t_{2} / t_{1}}$. T is the expiration time of compound options. The model is mainly used for the study of the investment decision of the complex logistics project.

2.5. The Logistics Project Evaluation Model under Fuzzy and Stochastic Environment. Similar to the building ways of the model under the random case, we still assume under the risk neutral world that asset value $A(t)$ follows a fuzzy differential equation; thus, we conclude the logistics project European call option pricing formula under the fuzzy and stochastic circumstances [5]:

$$
\begin{aligned}
\max & (0, A(T)) \\
= & \exp [-r(T-t)] E\left[\left(A(T)-K_{1}\right)^{+}\right] \\
= & \exp [-r(T-t)] \\
& \times E\left[A(t) \exp \left\{\left(\alpha(T-t)+\sigma_{1} z_{1}\right)-K_{1}\right\}^{+}\right] \\
= & \exp [-r(T-t)] \\
& \times \int_{0}^{+\infty} \operatorname{Cr}\left\{A(t) \exp \left(\alpha(T-t)+\sigma_{1} z_{1}\right)\right. \\
= & A(t) \exp [-r(T-t)] \\
& \times \int_{K_{1} / A(t)}^{+\infty} \operatorname{Cr}\left\{\exp \left(\alpha(T-t)+\sigma_{1} z_{1}\right) \geq x\right\} d x \\
= & A(t) \exp [-r(T-t)] \\
& \times \int_{K_{1} / A(t)}^{+\infty}\left(\exp \left(\frac{\alpha \pi}{\sqrt{6} \sigma_{1}}\right)\right) \\
& \quad \times\left(\exp \left(\frac{\alpha \pi}{\sqrt{6} \sigma_{1}}\right)\right. \\
= & \left.\quad+\exp \frac{\pi \operatorname{In} x}{\sqrt{6} \sigma_{1}(T-t)}\right)^{-1} d x \\
& \times \int_{K_{1} / A(t)}^{+\infty} \quad 1 \times\left(1+\exp \left(\frac{\pi}{\sqrt{6} \sigma_{1}(T-t)}\right.\right. \\
& \times(\operatorname{In} x-\alpha(T-t))))^{-1} d x
\end{aligned}
$$

Similarly, we still can get fuzzy the European call option pricing formula:

$$
\begin{aligned}
& \max (0, I(t)) \\
& \quad=I(t) \exp [-r(T-t)]
\end{aligned}
$$




$$
\begin{aligned}
\times \int_{K_{2} / I(t)}^{+\infty} 1 \times(1+\exp ( & \frac{\pi}{\sqrt{6} \sigma_{2}(T-t)} \\
& \times(\operatorname{In} x-\beta(T-t))))^{-1} d x .
\end{aligned}
$$

Then, we get the flow formula of the net present value under fuzzy environment

$$
\begin{aligned}
& \Phi(t)=\exp [-r(T-t)] E\left[\left(A(T)-K_{1}\right)^{+}\right] \\
&-\exp [-r(T-t)] E\left[\left(I(T)-K_{2}\right)^{+}\right]-I_{0} \\
&= A(t) \exp [-r(T-t)] \\
& \times \int_{K_{1} / A(t)}^{+\infty} 1 \times\left(1+\exp \left(\frac{\pi}{\sqrt{6} \sigma_{1}(T-t)}\right.\right. \\
&-I(t) \exp [-r(T-t)] \quad \times(\operatorname{In} x-\alpha(T-t))))^{-1} d x \\
& \times \int_{K_{2} / I(t)}^{+\infty} 1 \times\left(1+\exp \left(\frac{\pi}{\sqrt{6} \sigma_{2}(T-t)}\right.\right. \\
&\times(\operatorname{In} x-\beta(T-t))))^{-1} d x-I_{0}
\end{aligned}
$$

where $\alpha$ is the instantaneous fuzzy expected growth rate of $A(t), \beta$ is the instantaneous fuzzy expected growth rate of $I(t), \sigma_{1}$ is the instantaneous standard deviation of growth rate of $A(t), \sigma_{2}$ is instantaneous the standard deviation of growth rate of $I(t), d z_{1}$ is the standard Liu process of $A(t)$, and $d z_{2}$ is the standard Liu process of $I(t)$. The investment necessary conditions for $A(T)-I(T)>0, I_{0}$ are the initial investment and $r$ is the risk-less interest rate.

\section{Analysis and Discussion}

(1) Compared with the stochastic and the fuzzy environment model, taking full account of variable fuzzy situation in real, for example, in the process of logistics project investment decision making, whether investors should continue, stop, or delay the implementation of the project investment options, it is difficult to determine an absolute only data. Then, through the technology of fuzzy simulation, we can solve this kind of problems (see [4]). Under the basis of fuzzy simulation, this model provides a better solution.

(2) By $\theta^{2}=\sigma_{1}^{2}-2 \rho_{12} \sigma_{1} \sigma_{2}+\sigma_{2}^{2}$, from the formulas of net present value, the NPV method is not affected by $\rho_{12}, \sigma_{1}$, $\sigma_{2}$, and $\theta$. In general, there is only $\theta$ in calculation formula of the real option net present value flow, but not the other three, but the value of $\theta$ varies with $\rho_{12}, \sigma_{1}$, and $\sigma_{2}$ due to the $\rho_{12}=\left(\sigma_{1}^{2}+\sigma_{2}^{2}-\theta^{2}\right) / 2 \sigma_{1} \sigma_{2}$ in the $\rho_{12}$ set conditions; we easily calculated the values of $\theta$ according to the $\sigma_{1}$ and $\sigma_{2}$ values, but sometimes the $\rho_{12}$ valuation is very difficult, it will become one of the difficulties of formula of a common option net present value in the practical application, because the formula of fuzzy option net present value removes the $\rho_{12}$ factor and uses MATLAB by computer, which can quickly calculate results, which greatly simplifies the operation and improves the efficiency. Of course, if we compare the results calculated by three methods with $A^{*}(T)=\left(\theta_{1} /\left(\theta_{1}-\right.\right.$ 1)) $K_{1}$ and $I^{*}(T)=\left(\theta_{2} /\left(\theta_{2}-1\right)\right) K_{2}$, we can test these calculation results from another side.

(3) The NPV method is a traditional valuation method. Its advantages are simple and convenient; the disadvantage is obvious, that is, no consideration of investment management flexibility. The method of simple fuzzy net present value flow considers the fuzzy environment, fluctuations rate of the same, or different of real option value evaluation model; the fluctuations rate are considered under fuzzy environment. Based on the compound option value evaluation model of logistics project, the model used for the study of complex logistics project investment decision and, above, major in the random, fuzzy circumstances. The situation with random and fuzzy is a relatively new model which conforms well to reality. Due to the space problem, below, we mainly select three cases and explain with numerical examples.

\section{Examples}

After the fuzzy simulation [4], assuming a logistics enterprise to assess needs to invest 15 Billion dollars on the development project. After the project is successful, it needs 3 years to invest 15 Billion dollars in order to develop the market. Assuming that $A(0)=30$ Billion dollars, and risk-free interest rate is $6 \%, m_{1}=0.02, m_{2}=0.02, \alpha=0.03, \beta=0.01, \lambda_{1}=$ $0.08, \lambda_{2}=0.09, \sigma_{1}=0.25, \sigma_{2}=0.20$, and $\vartheta=0.2$.

Asking: how about the investment decision of the company? According to the traditional methods of net present value assessment, the whole project net present value stream is

$$
\Phi(t)=A(t) e^{-\alpha(T-t)}-I(t) e^{-\beta(T-t)}-I_{0}
$$

We have

$$
\begin{aligned}
\Phi(t)= & 30 \exp (-0.03 \times 3) \\
& -15 \exp (-0.06 \times 3)-15 \\
\approx & 30 \times 0.9139-15 \times 0.8353-15 \\
= & -0.1125 .
\end{aligned}
$$


Under the random environment, we have

$$
\begin{aligned}
\Phi(t)= & A(t) e^{-\alpha(T-t)} N\left(d_{1}\right) \\
& -I(t) e^{-\beta(T-t)} N\left(d_{2}\right)-I_{0} \Phi(t) \\
= & 30 \exp (-0.03 \times 3) N\left(d_{1}\right) \\
& -15 \exp (-0.06 \times 3) N\left(d_{2}\right)-15 \\
\approx & 30 \times 0.9139 \times N(2.434) \\
& -15 \times 0.8353 \times N(2.0876)-15 \\
\approx & 0.1175 .
\end{aligned}
$$

Under the fuzzy environment, we have

$$
\begin{aligned}
& \max (0, A(T)) \\
& =A(0) \exp (-r T) \\
& \times \int_{K / A(0)}^{+\infty} 1 \times\left(1+\exp \left(\frac{\pi}{\sqrt{6} \sigma_{1} T}\right.\right. \\
& \left.\left.\times\left(\operatorname{In} x-\left(m_{1}+\alpha-\lambda_{1}\right) T\right)\right)\right)^{-1} d x \\
& -I(0) \exp (-r T) \\
& \times \int_{K / I(0)}^{+\infty} 1 \times\left(1+\exp \left(\frac{\pi}{\sqrt{6} \sigma_{2} T}\right.\right. \\
& \left.\left.\times\left(\operatorname{In} x-\left(m_{2}+\beta-\lambda_{2}\right) T\right)\right)\right)^{-1} d x-15
\end{aligned}
$$

$\approx 11.3784$

In order to calculate $\exp (-r T) \int_{-\infty}^{K} \operatorname{Cr}\{(A(T) \leq u)\} d u$, $\exp (-r T) \int_{-\infty}^{K} \operatorname{Cr}\{(I(T) \leq u)\} d u$, using MATLAB, we can get on the computer [5]:

$$
\begin{aligned}
& \text { syms } \mathrm{x} \\
& \mathrm{y}=30 * \exp (-0.06 * 3) \cdot /(1+\exp ((\log (\mathrm{x})+0.03 * 3) * \mathrm{pi} / \\
& (\operatorname{sqrt}(6) * 0.25 * 3)))^{\prime} ; \\
& \mathrm{f}=\text { quad }(\mathrm{y}, 15 / 30,10000) \\
& \mathrm{f}=32.3977 \\
& \text { syms } \mathrm{x} ; \\
& \mathrm{y}=15 * \exp (-0.06 * 3) \cdot /(1+\exp ((\log (\mathrm{x})+0.06 * 3) * \mathrm{pi} / \\
& (\operatorname{sqrt}(6) * 0.20 * 3)))^{\prime} ; \\
& \mathrm{f}=\mathrm{quad}(\mathrm{y}, 15 / 15,10000) \\
& \mathrm{f}=6.2193) .
\end{aligned}
$$

As can be seen from the above, according to the traditional NPV method, the logistics project cannot be invested, but according to the random and fuzzy method, this project should be invested. Therefore, during the assessment of the logistics project, if using the traditional NPV method, we will often miss a lot of investment opportunities, easily to underestimate the sequence of the value of investment projects, so the result is that good prospects of gain project will usually be rejected.

\section{Conclusions}

Logistics project investment has high uncertainty, irreversibility, and the fuzziness. The NPV method usually adopted, for not considering the investment opportunity option value, it cannot reflect the actual value of the future well. In reality, we often still have no accurate valuation or cannot expect logistics project net cash flow situation, and because of the objective factors, some logistics project variables can not use the exact data to estimate the actual situation. So, by the accurate values determining model input parameters, evaluation results often deviate from the actual. In this paper, using the real option method, in reviewing the traditional model, we establish and discuss the logistics project valuation models under fuzzy environment; these models are important basis when we make the logistics project evaluation under fuzzy environment. Of course, for its scope of application as well as the practical problems to be solved in different emphasis, each model has its own characteristics. Of course, these models are not negative on the traditional investment decision methods, but, under the original basis, a reasonable explanation of investment income of fuzziness is made, which further increases the scientific and rationality of the investment decision making. In reality, we may combine logistics project investment opportunities in the variety and complexity, using multiple models as well as from multiple perspectives analysis. Finally, as far as possible, we will not miss good project. On the further discussion and research of the logistics project evaluation, the fuzzy random variable for rough fuzzy as well as the fourth party logistics problem of real options are the next problems we will try our best to solve.

\section{References}

[1] E. S. Schwartz and M. Moon, "Rational Pricing of Internet Companies," Financial Analysts Journal, vol. 56, no. 3, pp. 62$75,2000$.

[2] S. Dayanik, "Optimal stopping of linear diffusions with random discounting," Mathematics of Operations Research, vol. 33, no. 3, pp. 645-661, 2008.

[3] C. Carlsson and R. Fullér, "A fuzzy approach to real option valuation," Fuzzy Sets and Systems, vol. 139, no. 2, pp. 297-312, 2003.

[4] B. Liu, "Fuzzy process, hybrid process and uncertain process," Journal of Uncertain Systems, vol. 2, no. 1, pp. 3-16, 2008.

[5] Z. F. Qin and X. Li, "Option pricing formula for fuzzy financial market," Journal of Uncertain Systems, vol. 2, no. 1, pp. 17-21, 2008.

[6] R. McDonald and D. Siegel, "The value of waiting to investment," Quarterly Journal of Economics, vol. 101, no. 4, pp. 707727, 1986. 
[7] A. K. Dixit and R. S. Pindyck, “The Option Approach to Capital Investment," Harvard Business Review, vol. 73, no. 3, pp. 105-115, 1995.

[8] R. C. Merton, “Theory of rational option pricing," Bell Journal of Economics and Management Science, vol. 4, no. 1, pp. 141-183, 1973.

[9] R. Geske, “The valuation of compound options," Journal of Financial Economics, vol. 7, no. 1, pp. 63-81, 1979. 

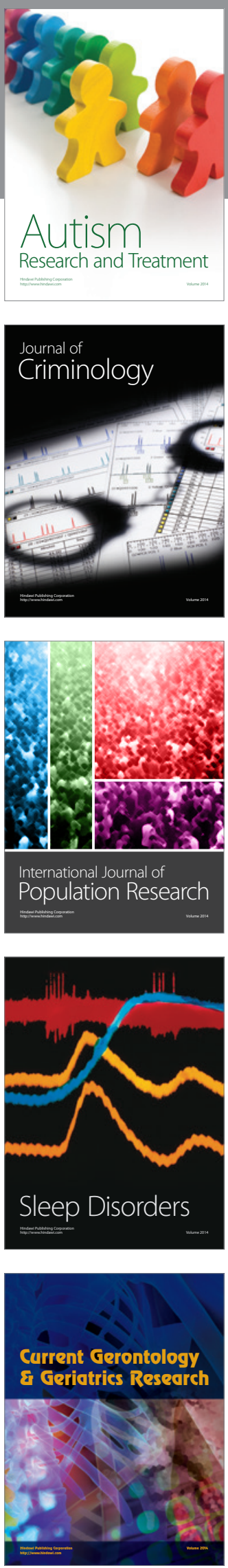
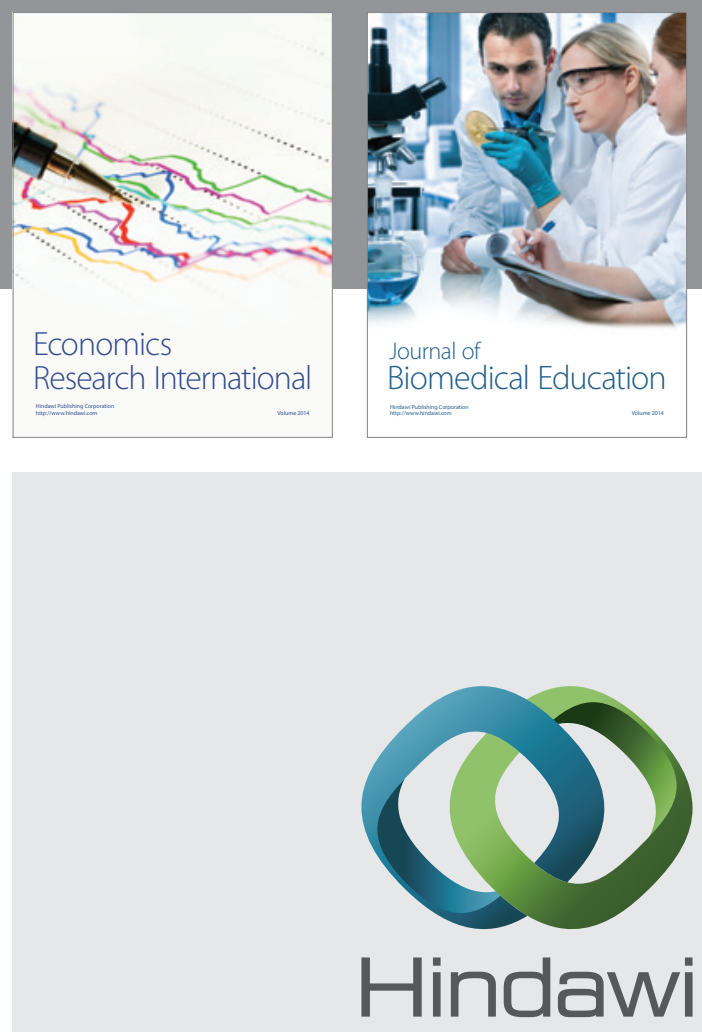

Submit your manuscripts at

http://www.hindawi.com
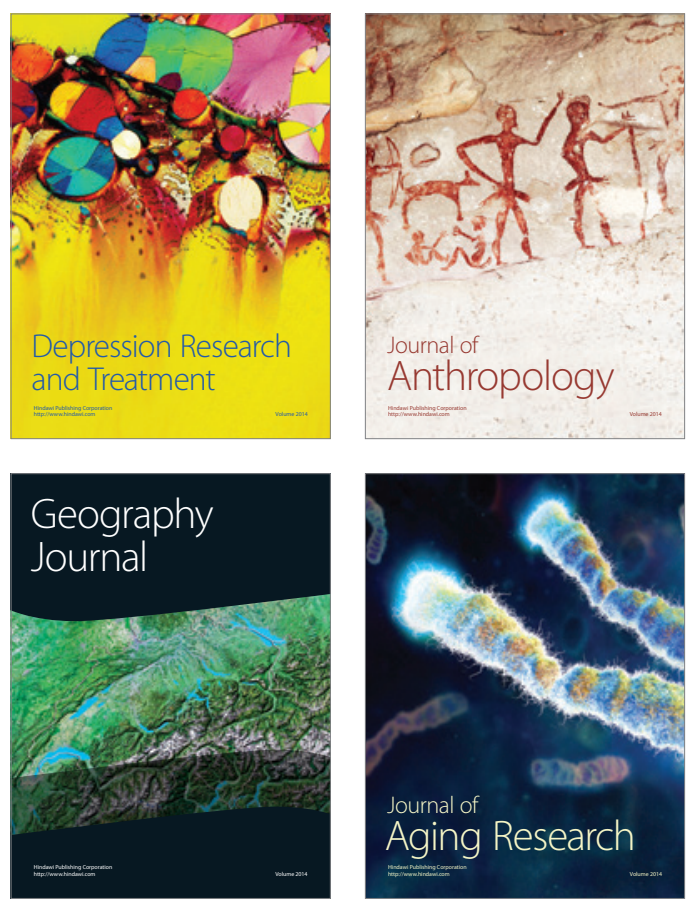
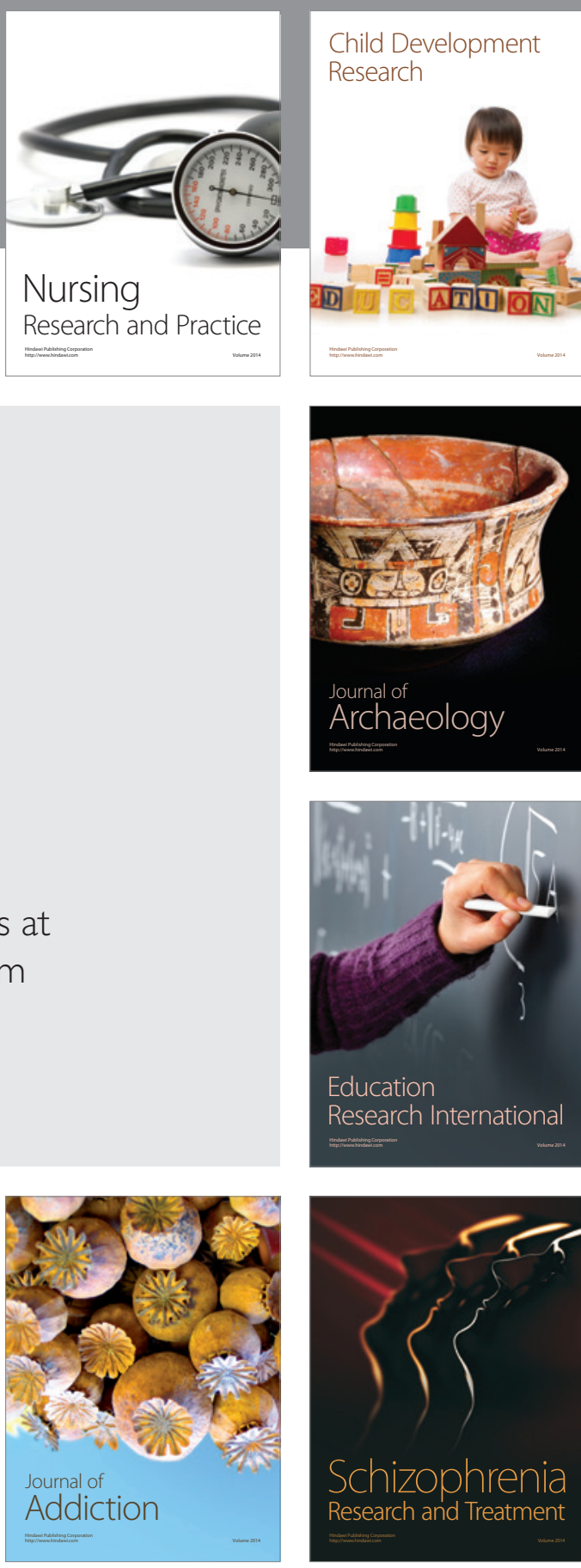

(D)
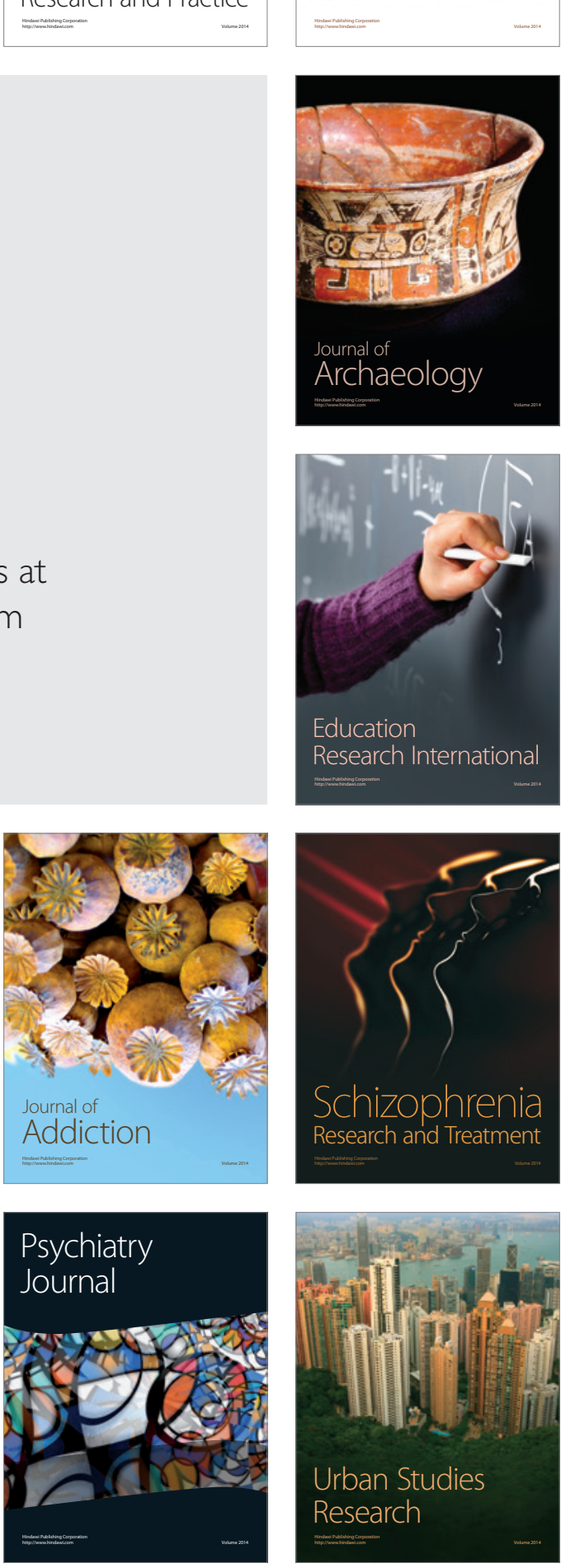